\title{
Detection of miR-29a in plasma of patients with lumbar spinal stenosis and the clinical significance
}

\author{
GENAI ZHANG ${ }^{1}$, WENPING ZHANG ${ }^{2}$, YU HOU $^{1}$, YINGCHUN CHEN ${ }^{1}$, JIPENG SONG ${ }^{1}$ and LIXIANG DING $^{1}$ \\ ${ }^{1}$ Department of Spinal Surgery, Beijing Shi Ji Tan Hospital, Capital Medical University, Beijing 100038; \\ ${ }^{2}$ School of Public Health, Shanxi Medical University, Taiyuan, Shanxi 030001, P.R. China
}

Received October 9, 2017; Accepted March 1, 2018

DOI: $10.3892 / \mathrm{mmr} .2018 .8956$

\begin{abstract}
The present study aimed to detect miR-29a expression in the plasma of patients with lumbar spinal stenosis (LSS) and to investigate the clinical significance. A total of 30 patients with LSS, 27 patients with lumbar intervertebral disc herniation (LDH), 27 healthy people and 7 patients that had succumbed to mortality were involved in the present study for specimen collection. Expression levels of miR-29a in plasma and intervertebral disc tissue were detected by reverse transcription-quantitative polymerase chain reaction analysis. Plasma expression levels of matrix metalloproteinase 9 (MMP9) and a disintegrin and metalloproteinase with thrombospondin motifs 5 (ADAMTS5) were detected ELISA. The expression levels of MMP9 and ADAMTS5 protein were detected by western blotting. Pearson correlation analysis was used to analyze the correlations between the expression levels of microRNA (miR)-29a, MMP9 and ADAMTS5. Receiver operating characteristic curve analysis was used to analyze the possibility of the use of miR-29a as a biomarker of LSS. The expression levels of miR-29a in plasma and intervertebral disc tissue of patients with LSS were significantly lower in patients with LSS compared with in patients with LDH, as well as healthy controls. Conversely, the protein expression levels of MMP9 and ADAMTS5 were significantly higher in patients with LSS compared with patients with LDH, as well as healthy controls. The expression levels of miR-29a was negatively correlated with the expression levels of MMP9 and ADAMTS5. In addition, miR-29a demonstrated low temperature sensitivity and high freeze-thaw stability, and may be used to accurately diagnose LSS. Therefore, miR-29a may be considered to be a potential biomarker of LSS.
\end{abstract}

Correspondence to: Dr Lixiang Ding, Department of Spinal Surgery, Beijing Shi Ji Tan Hospital, Capital Medical University, 10 Tieyi Road, Beijing 100038, P.R. China

E-mail:1fqm1707@163.com

Key words: microRNA-29a, lumbar spinal stenosis, biomarker

\section{Introduction}

Lumbar spinal stenosis (LSS) refers to the lower or buttock extremity pain caused by the reduced space for vascular and neural elements in the lumbar spine (1). LSS causes severe pain that can seriously affect the quality of life of patients, particularly in elderly patients with risks falling, disability and depression (2). In addition, the lack of satisfactory treatment outcomes and the costs of surgery places heavy economic and psychological burden on patients and their families (3). LSS mainly affects patients older than 65 years. It has been reported that the proportion of people older than 65 years will increase from 8 to $14 \%$ in next several decades, indicating a potential increase in incidence rate of LSS (4). A variety of methods, including imaging techniques, have been developed to diagnose LSS; however, the application of most of those methods is limited by unsatisfactory accuracy (5). Therefore, the development of diagnostic methods than can be used to accurately identify LSS is required.

The pathogenesis of LSS remains to be fully elucidated. As a group small noncoding RNAs containing 22-24 nucleotides, microRNAs (miRs) have been observed to participate in a variety of biological processes, including cell proliferation, differentiation and fibrosis (6-8). Previous studies have demonstrated that the development of LSS is associated with the function of various miRNAs $(9,10)$. As a member of miR-29 family, miR-29a has been reported to be involved in the deterioration of synovitis in patients with end-stage knee arthritis (11); however, the role of miR-29a in the development of LSS requires further investigation.

In the present study, the expression of miR-29a in plasma and intervertebral disc tissue of patients with LSS and lumbar intervertebral disc herniation (LDH) were detected. In addition, the possibility of the application of miR-29a as a biomarker of LSS was investigated.

\section{Materials and methods}

Patients. A total of 30 patients with LSS, including 21 males and 9 females were selected in the Beijing Shi Ji Tan Hospital (Beijing, China) between January 2015 and January 2017; the average age of the patients was $60 \pm 4.3$-years-old. Simultaneously, 27 patients with LDH, including 20 males and 7 females were also selected, and average age of those patients 
was $57 \pm 6.1$-years-old. In addition, 27 healthy individuals with similar age distribution (19 males and 8 females with a mean age of $56 \pm 7.7$ years) were selected to collected plasma samples, and 7 patients that had succumbed to mortality (5 males and 2 females with a mean age of $58 \pm 5.2$ years) were selected to collect normal intervertebral disc tissue. All patients were diagnosed by pathological examination and medical imaging. Patients with lumbar spondylolisthesis, cancer, infection, hypertension, heart disease and diabetes were excluded. All patients with LSS underwent laminectomy for decompression and surgical resection of intervertebral disc tissue. The present study was approved by the Ethics Committee of Beijing Shi Ji Tan Hospital. All patients provided written informed consent.

Specimen collection. Blood $(5 \mathrm{ml})$ was extracted from middle vein of the elbow of each participant. Red blood cells were removed by centrifugation at $106 \mathrm{x} \mathrm{g}$ for $20 \mathrm{~min}$ at room temperature, and plasma was transferred to $0.5 \mathrm{ml}$ sterile Eppendorf tubes (Eppendorf, Hamburg, Germany) and stored at $-20^{\circ} \mathrm{C}$ until use. Intervertebral disc tissue was collected from patients with LSS and LDH. Normal intervertebral disc tissue was collected from patients without lumbar diseases that had succumbed to mortality. Tissues were washed with saline and stored at $-80^{\circ} \mathrm{C}$ prior to use.

Enzyme-linked immunosorbent assay (ELISA). Human MMP-9 Quantikine ELISA kit DMP900 (R\&D Systems, Inc., Minneapolis, MN, USA) and Human ADAMTS5 DuoSet ELISA kit (R\&D Systems, Inc.) were used according to the manufacture's protocols.

Reverse transcription-quantitative polymerase chain reaction (RT-qPCR). Intervertebral disc tissue was sectioned into pieces and ground in liquid nitrogen. TRIzol (Thermo Fisher Scientific, Inc., Waltham, MA, USA) was used to extract RNA from plasma and intervertebral disc tissue. RNA samples were dissolved in diethyl pyrocarbonate water. Only RNA samples with an optical density ratio $(260 / 280 \mathrm{~nm})$ between 1.8 and 2.0 were used. RT was performed to synthesize cDNA using Oligo (dT) 15 (Sangon Biotech Co., Ltd., Shanghai, China) and AMV reverse transcriptase (Gibco; Thermo Fisher Scientific, Inc.). Reaction conditions were: $25^{\circ} \mathrm{C}$ for $5 \mathrm{~min}, 55^{\circ} \mathrm{C}$ for $45 \mathrm{~min}$ and $80^{\circ} \mathrm{C}$ for $20 \mathrm{~min}$. SYBR ${ }^{\circledR}$ Green Real-Time PCR Master Mix (Thermo Fisher Scientific, Inc.) was used to prepare the qPCR reaction system. Primers used were: miR-29a forward, 5'-ATAGGATCCCGACCTTCTGTGACCCCTTA-3', reverse, 5'-CGCAAGCTTACCACATGCAATTCAGGTCA-3' and GAPDH forward, 5'-GAGTCAACGGATTTGGTCGT-3' and reverse, 5'-TTGATTTTGGAGGGATCTCG-3'. qPCR was performed on a Bio-Rad iCycler (Bio-Rad Laboratories, Inc., Hercules, CA, USA). The reaction conditions were: $94^{\circ} \mathrm{C}$ for $2 \mathrm{~min}$, followed by 40 cycles of $94^{\circ} \mathrm{C}$ for $10 \mathrm{sec}, 55^{\circ} \mathrm{C}$ for $30 \mathrm{sec}$ and $72^{\circ} \mathrm{C}$ for $25 \mathrm{sec} . \mathrm{Cq}$ values were processed using $2^{-\Delta \Delta \mathrm{Cq}}$ method (12), and relative expression levels of miR-29a were normalized to that of the endogenous control, GAPDH.

Western blotting. Protein was extracted from intervertebral disc tissue using RIPA buffer (Thermo Fisher Scientific, Inc.). A Bicinchoninic Acid protein method was used to determine the concentration of protein; $20 \mu \mathrm{g}$ of protein from

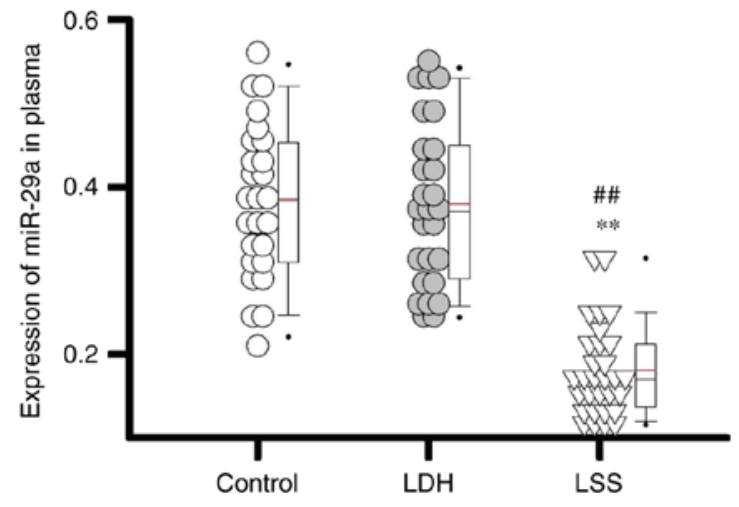

Figure 1. Expression levels of miR-29a in the plasma of various patient groups. Triangles and black dots represent the ROC curve of LDH and LSS, respectively. The gray lines are lines of identity. ${ }^{* *} \mathrm{P}<0.01$, compared with the control group. ${ }^{\#} \mathrm{P}<0.01$, compared with patients with $\mathrm{LDH}$. Control, healthy patients; LDH, lumbar intervertebral disc herniation; LSS, lumbar spinal stenosis; miR, microRNA.

each sample was subjected to $10 \%$ SDS-PAGE, followed by transfer onto polyvinylidene difluoride membranes (Bio-Rad Laboratories, Inc.). Membranes were blocked with 5\% skimmed milk at room temperature for $2 \mathrm{~h}$. Following washing with tris-buffered saline with Tween-20 (0.3\%), membranes were incubated with primary antibodies including anti-matrix metalloproteinase 9 (MMP9; 1:1,000; cat. no. EP1255Y; Abcam, Cambridge, UK), anti-a disentigrin and metalloproteinase with thrombospondin motifs 5 (ADAMTS5; $1: 1,000$; cat. no. ab41037; Abcam), and anti- $\beta$-actin $(1: 1,000$; cat. no. ab8227; Abcam) overnight at $4^{\circ} \mathrm{C}$. Following washing with tris-buffered saline with Tween-20 (0.3\%), membranes were incubated with goat anti-rabbit immunoglobulin G horseradish peroxidase secondary antibody (cat. no. sc-2004; Santa Cruz Biotechnology, Inc., Dallas, TX, USA) at $37^{\circ} \mathrm{C}$ for $1 \mathrm{~h}$. An enhanced chemiluminescence assay was performed according to the protocols of the Pierce ECL Western Blotting Substrate (Pierce; Thermo Fisher Scientific, Inc.) and iBright Imaging Systems (Thermo Fisher Scientific, Inc.) was used to visualize the bands. Data was analyzed by Image $\mathbf{J}$ version 1.48 (National Institutes of Health, Bethesda, MD, USA).

Determination of the stability of Plasma miR-29a. Plasma samples were kept at room temperature or at $4^{\circ} \mathrm{C}$, and sampling was performed 2, 4, 6, 8, 10 and $12 \mathrm{~h}$ later. Plasma samples were also subjected to freeze-thaw cycles $\left(-80^{\circ} \mathrm{C}\right.$ for $20 \mathrm{~min}$ and room temperature for $20 \mathrm{~min}$ ). The amount of $\mathrm{miR}-29 \mathrm{a}$ retained in plasma samples was measured by RT-qPCR.

Statistical analysis. SPSS software version 19.0 (IBM Corp., Armonk, NY, USA) was used to analyze the data. Each experiment was performed 3 times and data were expressed as mean \pm standard deviation. Comparisons between groups were performed by an unpaired Student's t-test, and comparisons among multiple groups were performed by one way-analysis of variance followed by the Least Significant Difference test. Correlations between the expression levels of miR-29 and the expression levels of MMP9 and ADAMTS5, and the correlation between expression levels in plasma and intervertebral disc tissue were performed by Spearman's correlation 
A

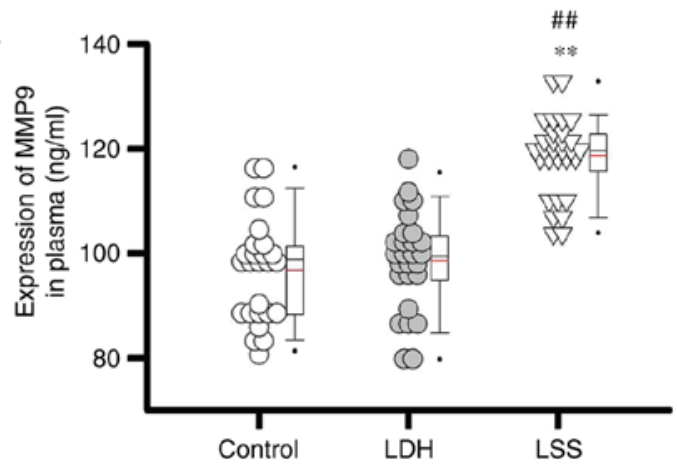

B

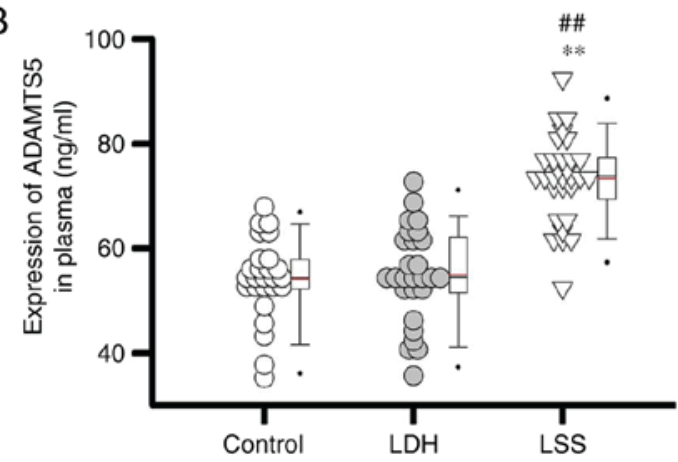

Figure 2. Comparison of plasma levels of (A) MMP9 and (B) ADAMTS5 between different patient groups. Triangles and black dots represent the ROC curve of LDH and LSS, respectively. The gray lines are lines of identity. ${ }^{* *} \mathrm{P}<0.01$, compared with the control group. ${ }^{\# \#} \mathrm{P}<0.01$, compared with patients with $\mathrm{LDH}$. ADAMTS5; a disintegrin and metalloproteinase with thrombospondin motifs 5; control, healthy patients; LDH, lumbar intervertebral disc herniation; LSS, lumbar spinal stenosis; MMP9, matrix metalloproteinase 9.
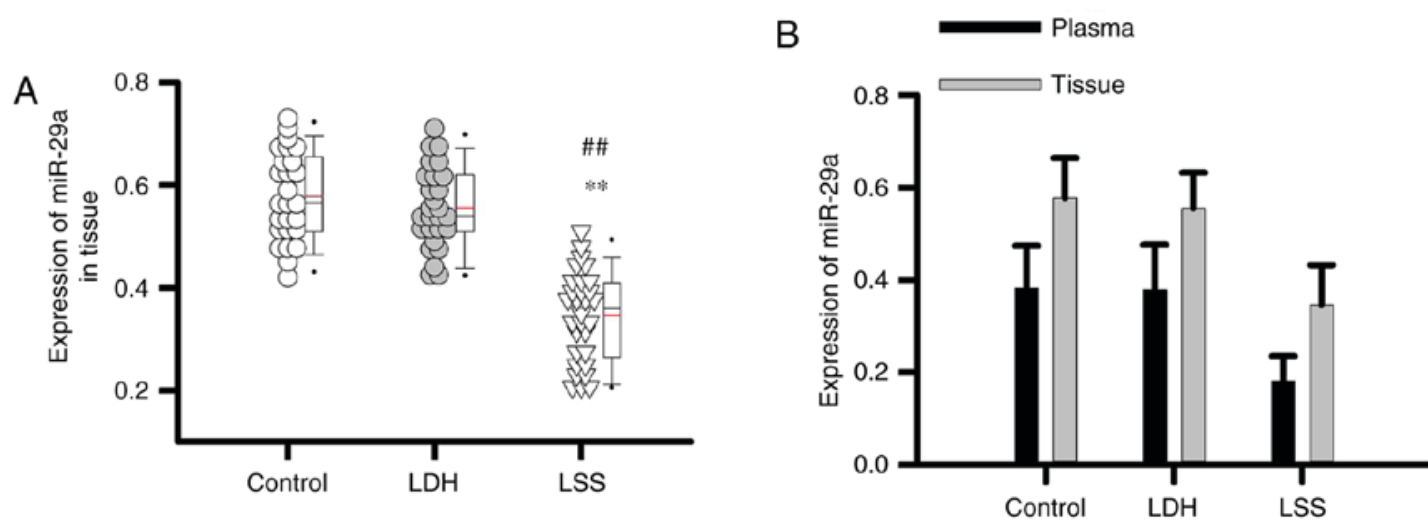

Figure 3. Expression levels of miR-29a of various patient groups. (A) Expression levels of miR-29a in intervertebral disc tissue of different groups of patients; (B) comparison of the expression levels of miR-29a in plasma and intervertebral disc tissue. Triangles and black dots represent the ROC curve of LDH and LSS, respectively. The gray lines are lines of identity. $\mathrm{P}<0.01$, compared with the control group; ${ }^{*} \mathrm{P}<0.01$, compared with patients with LDH. Control, healthy patients; LDH, lumbar intervertebral disc herniation; LSS, lumbar spinal stenosis; miR, microRNA.

coefficient analysis. Receiver operating characteristic (ROC) curve analysis was performed to evaluate the diagnostic value of miR-29 for LSS and LDH. P $<0.05$ was considered to indicate a statistically significant difference.

\section{Results}

Expression levels of miR-29a in plasma of different groups of patients. As presented in Fig. 1, the expression levels of miR-29a were significantly lower in patients with LSS compared with in patients with $\mathrm{LDH}$ and healthy individuals (both $\mathrm{P}<0.01$ ). The data of the present study suggested that the expression levels of miR-29a were downregulated in patients with LSS.

Comparison of plasma levels of MMP9 and ADAMTS5 between different patient groups. miR-29a expression is positively (13) or negatively (14) correlated with the expression of MMP9 in a variety of disease models; however, the effects of miR-29a expression on MMP9 in LSS require further investigation. A recent study revealed that miR-29a targeted ADAMTS5 to reduce its expression levels in patients with knee osteoarthritis (11). Therefore, the plasma expression levels of
MMP9 and ADAMTS5 were detected in a variety of patient groups. The results of the present study revealed that plasma expression levels of MMP9 (Fig. 2A) and ADAMTS5 (Fig. 2B) in patients with LSS were significantly higher compared with patients with LDH and healthy controls (all $\mathrm{P}<0.01$ ). The data suggested that the expression levels of miR-29a were inhibited in patients with LSS, which in turn may have upregulated plasma expression levels of MMP9 and ADAMTS5.

Comparison of the expression of miR-29a in intervertebral disc tissue of different groups of patients. The expression levels of miR-29a in intervertebral disc tissue of patients with LSS were significantly lower than that of patients with $\mathrm{LDH}$, as well as healthy controls $(\mathrm{P}<0.01$; Fig. 3A). In addition, the expression levels of miR-29a in plasma revealed similar expression profiles to that in intervertebral disc tissue (Fig. 3B). The data indicated that the plasma expression levels of miR-29a may reflect its expression in intervertebral disc tissue.

Comparison of MMP9 and ADAMTS5 expression levels in intervertebral disc tissue of different groups of patients. The expression levels of MMP9 (Fig. 4A) and ADAMTS5 (Fig. 4B) in intervertebral disc tissue of patients with LSS were 
A

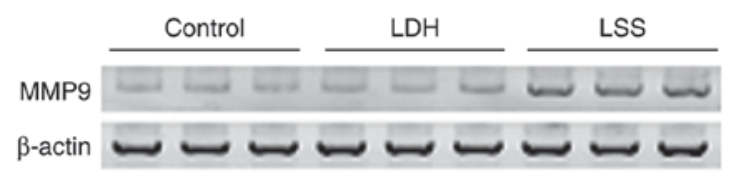

B

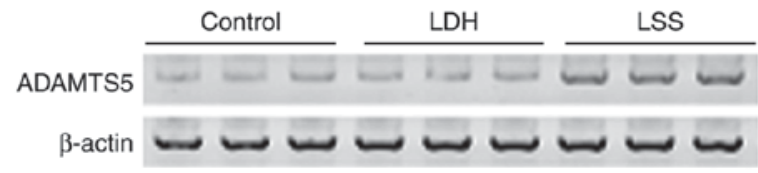

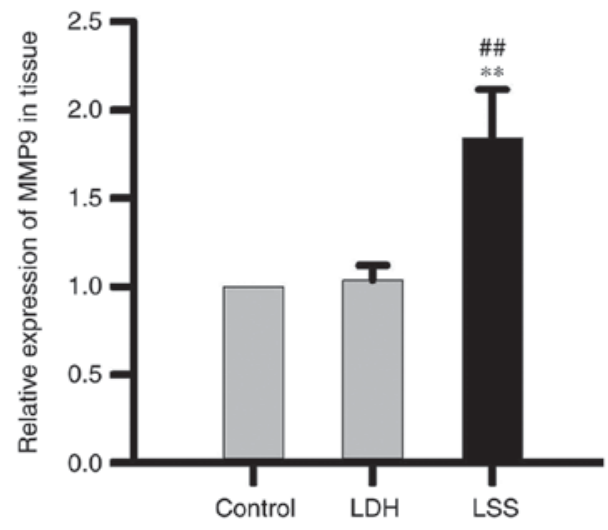

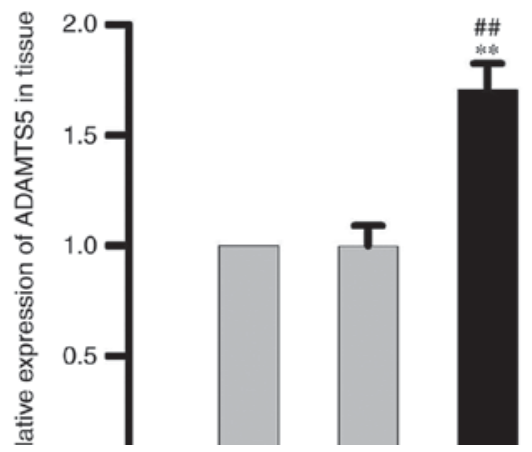

Figure 4. Comparison of MMP9 and ADAMTS5 expression levels in intervertebral disc tissue of various patient groups. (A) Expression levels of MMP9 and ADAMTS5 in intervertebral disc tissue of various patient groups; (B) Expression levels of ADAMTS5 in intervertebral disc tissue of various patient groups. ${ }^{* *} \mathrm{P}<0.01$, compared with the control group. ${ }^{\sharp \#} \mathrm{P}<0.01$, compared with patients with LDH. ADAMTS5; a disintegrin and metalloproteinase with thrombospondin motifs 5; control, healthy patients; LDH, lumbar intervertebral disc herniation; LSS, lumbar spinal stenosis; MMP9, matrix metalloproteinase 9.

A

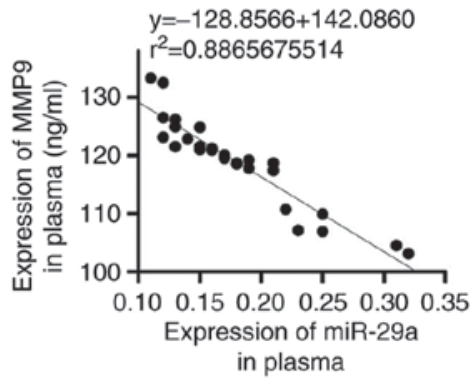

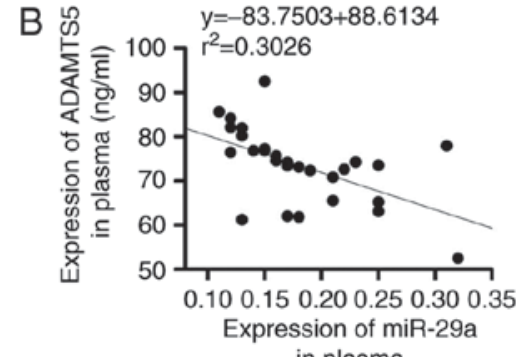

in plasma
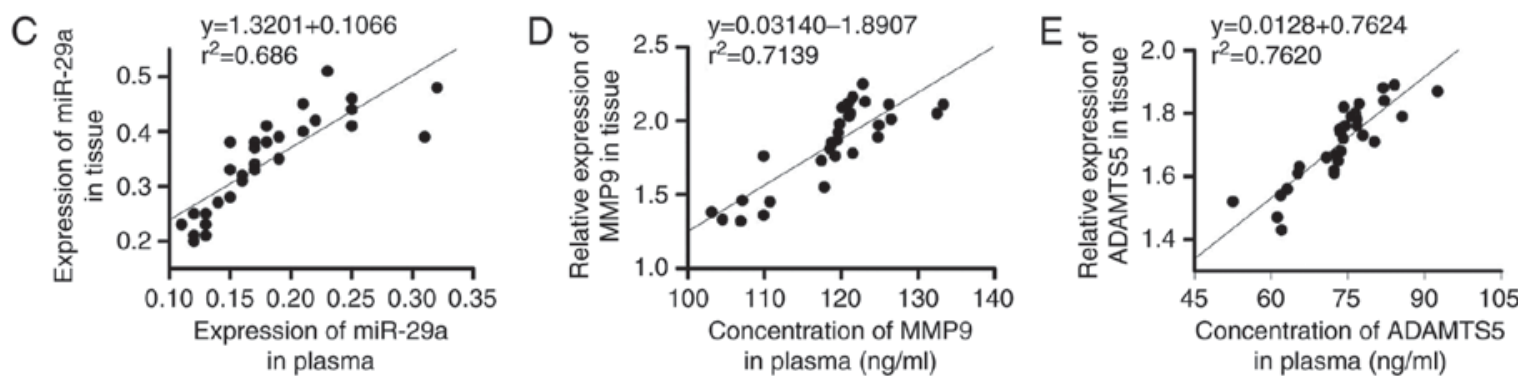

Figure 5. Correlations between the expression levels of miR-29, MMP9 and ADAMTS5, and correlation between their expression levels in plasma and intervertebral disc tissue of LSS patients. (A) Correlation between the expression levels of miR-29 and MMP9. (B) Correlation between the expression levels of miR-29 and ADAMTS5. (C) Correlation between the expression levels of miR-29 in plasma and intervertebral disc tissue. Correlation between the expression levels of (D) MMP9 and (E) ADAMTS5 in plasma and intervertebral disc tissue. $\mathrm{y}$, the binary equation representing the distributions of values; ADAMTS5; a disintegrin and metalloproteinase with thrombospondin motifs 5; MMP9, matrix metalloproteinase 9.

significantly higher compared with patients with LDH, as well as healthy controls. The data suggested that the expression levels of MMP9 and ADAMTS5 were increased in intervertebral disc tissue of patients with LSS.
Correlations between the expression levels of $m i R-29$ and the expression levels of MMP9 and ADAMTS5, and the correlation between expression levels in plasma and intervertebral disc tissue. The expression levels of miR-29 were negatively 
correlated with that of MMP9 (Fig. 5A) and ADAMTS5 (Fig. 5B). In addition, the expression levels of miR-29 (Fig. 5C), MMP9 (Fig. 5D) and ADAMTS5 (Fig. 5E) in plasma were positively correlated with their expression levels in intervertebral disc tissue of LSS patients $(\mathrm{P}<0.05)$.

ROC curve analysis of the prediction of LSS and LDH by $m i R-29$. ROC curve analysis was performed to investigate the diagnostic value of plasma miRNA-29 for LSS and LDH. The area under the curve of miR-29 associated with LSS was 0.97 , while the area under the curve of miR-29 in predicting LDH was only 0.51 (Fig. 6). The findings of the present study indicated that miR-29 may be used to accurately diagnose LSS.

Stability of miR-29a in the plasma of patients with LSS. The present study revealed that miR-29a may be used to accurately diagnose LSS but not LDH; however, the application of miR-29a as a biomarker of LSS also requires high RNA stability. Therefore, temperature sensitivity and freeze-thaw stability of miR-29a were detected by RT-qPCR. The results demonstrated that miR-29a under room temperature showed similar stability to that at $4^{\circ} \mathrm{C}$ (Fig. 7A). In addition, miR-29a in plasma also exhibited high freeze-thaw stability (Fig. 7B), and about $50 \%$ of the RNA was retained in plasma after 5 cycles of freeze-thaw. These findings suggested that miR-29a may be easily detected under a variety of conditions, which in turn may reduce the cost and the requirement of equipment for the diagnosis test. Therefore, miR-29a may be considered to be a promising biomarker for LSS.

\section{Discussion}

Development of LSS is a complex process with various internal and external factors involved. At present, pathogenesis of LSS remains to be investigated; however, various clinical and experimental studies have reported that overweight and obese individuals usually have high risk of LSS due to relatively high incidences of inflammatory responses $(15,16)$. Inflammation is closely associated with the progression of LSS (16). Angiopoietin-like protein 2 can accelerate the progression of lumbar spinal canal stenosis by promoting inflammatory responses via the activation of interleukin (IL)-6, which serves a role as both an proinflammatory and anti-inflammatory factor (17). miRNAs constitute a group of small noncoding RNAs that serve pivotal roles in a variety biochemical and physiological processes. Involvement of miRNAs in the progression of intervertebral disc-associated diseases has also been reported recently $(18,19)$. miRNA-146a, as a member of the miRNA-146 family, was reported to limit inflammatory responses by inhibiting the expression of IL-10 in intervertebral discs (18). Conversely, miR-515 and -194 were demonstrated to promote the development of intervertebral disc degeneration by interrupting the biosynthesis of chondroitin sulfate in humans (19). In the study of LSS, $\mathrm{Xu}$ et al (10) reported that overexpression of collagens I and III induced by treatment with inhibitors of MMP reduced the expression levels of miRNA-221, which in turn promoted the progression of hypertrophy of ligamentum flavum in patients with LSS.

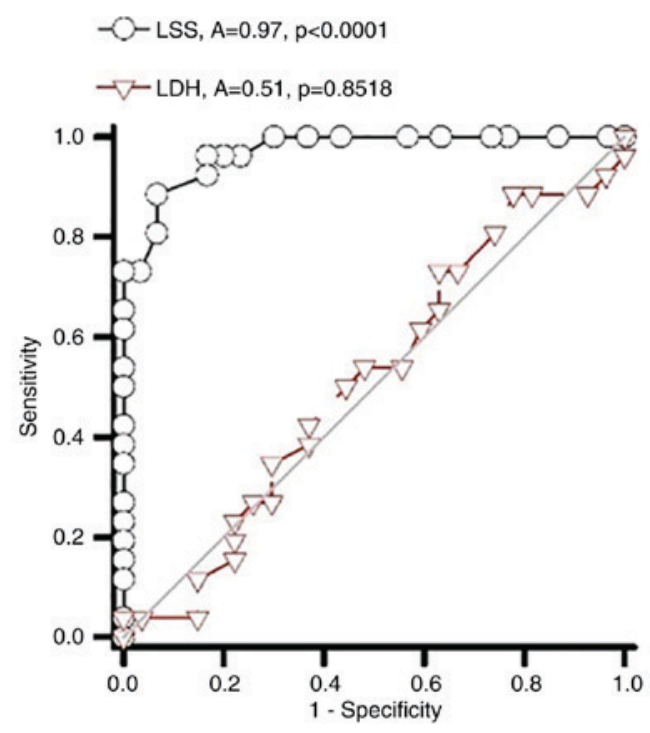

Figure 6. Receiver operating characteristic curve analysis of the prediction of LSS and LDH via miR-29 expression. A, area under the curve.
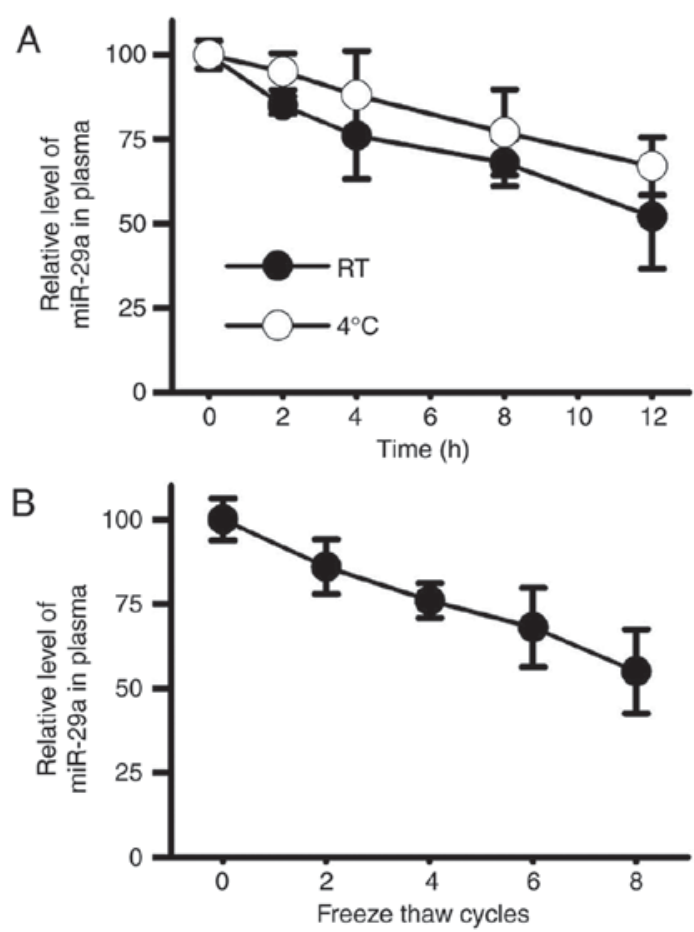

Figure 7. Temperature sensitivity and freeze-thaw stability of miR-29a in plasma derived from LSS patients. (A) Temperature sensitivity of miR-29a in plasma; (B) Freeze-thaw stability of miR-29a in plasma. Relative level of miR-29a retained in plasma was normalized to the level at the beginning of the experiment. miR, microRNA; RT, room temperature.

As a member of miR-29 family, miR-29a was revealed to be involved in the development of a variety diseases. In the study of cerebellar alterations in mice, Papadopoulou et al (20) reported that miR-29a together with miR-29b-1 caused the ataxic features of this disease. The expression levels of miR-29a were significantly upregulated in serum of patients with acute graft-vs-host disease; increased expression levels of miR-29a were suggested to activate dendritic cells via Toll-like 
receptor (TLR) 8 and TLR 7 (21). However, to the best of the authors' knowledge, expression profile and functionality of miR-29a in the pathogenesis of LSS have yet to be reported. In the present study, the expression levels miR-29a were observed to be significantly lower in patients with LSS than in patients with LDH, as well as healthy controls, indicating that the expression of miR-29a may be particularly downregulated and correlated with the progression of LSS. ADAMTSs and MMPs are main factors involved in the process of disc degeneration and breakdown of the extracellular matrix (22). miR-29a can negatively regulate the expression of MMP9 and ADAMTS5 (11). In the present study, the expression levels of MMP9 and ADAMTS5 were observed to be significantly higher in patients with LSS than in patients with LDH, as well as healthy controls. In addition, the expression levels of miR-29a were reported to be negatively correlated with that of MMP9 and ADAMTS5. The results of the present study suggest that reduced expression of miR-29a may upregulate the expression of MMP9 and ADAMTS5, which in turn may promote the progression of this LSS.

Due to the particular expression profile of miR-29a in numerous pathological processes, miR-29a has been applied as a biomarker for the diagnosis of a variety of human diseases. In the study of colorectal cancer, Brunet Vega et al (23) reported that the expression levels of miR-29a were significantly increased in patients with stage III colorectal cancer, and expression levels of miR-29a may be used to accurately predict this disease. In addition, the expression levels of numerous miRNAs were detected to be upregulated in patients with hypertrophic cardiomyopathy; only circulating miR-29a can be used to sensitively and accurate diagnose both hypertrophy and fibrosis (24). In the present study, the expression levels of miR-29a in plasma reflected its expression profile within intervertebral disc tissue. Therefore, plasma expression levels of miR-29a may be employed to predict LSS; plasma expression levels of miR-29a may be used to accurately predict LSS but not LDH. In addition, miR-29a was also demonstrated to exhibit low temperature sensitivity and high freeze-thaw stability. The findings of the present study suggested that miR-29a may serve as a reliable biomarker for the diagnosis of LSS.

In conclusion, the expression levels of miR-29a were particularly reduced in patients with LSS, which in turn led to the increased expression levels of MMP9 and ADAMTS5, resulting in the progression of LSS. In addition, plasma expression levels of miR-29a, which exhibited low temperature sensitivity and high freeze-thaw stability, may be applied to accurately diagnose LSS.

\section{Acknowledgements}

Not applicable.

\section{Funding}

No funding was received.

\section{Availability of data and materials}

All data generated or analyzed during this study are included in this published article.

\section{Authors' contributions}

GZ and LD designed the experiments. GZ, WZ and YH performed the experiments. GZ, YC, JS and LD analyzed the data. $L D$ wrote the manuscript. All authors read the manuscript.

\section{Ethics approval and consent to participate}

The present study was approved by the Ethics Committee of Beijing Shi Ji Tan Hospital. All patients provided written informed consent.

\section{Consent for publication}

All participants signed informed consent.

\section{Competing interests}

The authors declare that they have no competing interests.

\section{References}

1. Kreiner DS, Shaffer WO, Baisden JL, Gilbert TJ, Summers JT, Toton JF, Hwang SW, Mendel RC and Reitman CA; North American Spine Society: An evidence-based clinical guideline for the diagnosis and treatment of degenerative lumbar spinal stenosis (update). Spine J 13: 734-743, 2013.

2. Backstrom KM, Whitman JM and Flynn TW: Lumbar spinal stenosis-diagnosis and management of the aging spine. Man Ther 16: 308-317, 2011.

3. Lucio JC, VanConia RB, DeLuzio KJ, Lehmen JA, Rodgers JA and Rodgers W: Economics of less invasive spinal surgery: An analysis of hospital cost differences between open and minimally invasive instrumented spinal fusion procedures during the perioperative period. Risk Manag Healthc Policy 5: 65-74, 2012.

4. Bressler HB, Keyes WJ, Rochon PA and Elizabeth B: The prevalence of low back pain in the elderly: A systematic review of the literature. Spine (Phila Pa 1976) 24: 1813-1819, 1999.

5. de Schepper EI, Overdevest GM, Suri P, Peul WC, Oei EH, Koes BW, Bierma-Zeinstra SM and Luijsterburg PA: Diagnosis of lumbar spinal stenosis: An updated systematic review of the accuracy of diagnostic tests. Spine (Phila Pa 1976) 38: E469-E481, 2013.

6. Jiang X, Tsitsiou E, Herrick SE and Lindsay MA: MicroRNAs and the regulation of fibrosis. FEBS J 277: 2015-2021, 2010.

7. Shenoy A and Blelloch RH: Regulation of microRNA function in somatic stem cell proliferation and differentiation. Nat Rev Mol Cell Biol 15: 565-576, 2014.

8. Baumjohann D and Ansel KM: MicroRNA-mediated regulation of $T$ helper cell differentiation and plasticity. Nat Rev Immunol 13: 666-678, 2013.

9. Chen J, Liu Z, Zhong G, Qian L, Li Z, Qiao Z, Chen B and Wang H: Hypertrophy of ligamentum flavum in lumbar spine stenosis is associated with increased miR-155 level. Dis Markers 2014: 786543, 2014.

10. Xu YQ, Zhang ZH, Zheng YF and Feng SQ: MicroRNA-221 regulates hypertrophy of ligamentum flavum in lumbar spinal stenosis by targeting TIMP-2. Spine (Phila Pa 1976) 41: 275-282, 2016.

11. Ko JY, Lee MS, Lian WS, Weng WT, Sun YC, Chen YS and Wang FS: MicroRNA-29a counteracts synovitis in knee osteoarthritis pathogenesis by targeting VEGF. Sci Rep 7: 3584, 2017.

12. Livak KJ and Schmittgen TD: Analysis of relative gene expression data using real-time quantitative PCR and the 2(-Delta Delta C(T)) method. Methods 25: 402-408, 2001.

13. Han W, Han Y, Liu X and Shang X: Effect of miR-29a inhibition on ventricular hypertrophy induced by pressure overload. Cell Biochem Biophys 71: 821-826, 2015.

14. Chen Q, Yin D, Zhang Y, Yu L, Li XD, Zhou ZJ, Zhou SL, Gao DM, Hu J, Jin C, et al: MicroRNA-29a induces loss of 5-hydroxymethylcytosine and promotes metastasis of hepatocellular carcinoma through a TET-SOCS1-MMP9 signaling axis. Cell Death Dis 8: e2906, 2017. 
15. Knutsson B, Sandén B, Sjödén G, Järvholm B and Michaëlsson K: Body mass index and risk for clinical lumbar spinal stenosis: A cohort study. Spine (Phila Pa 1976) 40: 1451-1456, 2015.

16. Fujita N, Hosogane N, Hikata T, Iwanami A, Watanabe K, Shiono Y, Okada E, Ishikawa M, Tsuji T, Shimoda M, et al: Potential Involvement of obesity-associated chronic inflammation in the pathogenesis of idiopathic spinal epidural lipomatosis. Spine (Phila Pa 1976) 41: E1402-E1407, 2016.

17. Nakamura T, Okada T, Endo M, Nakamura T, Oike $Y$ and Mizuta $\mathrm{H}$ : Angiopoietin-like protein 2 promotes inflammatory conditions in the ligamentum flavum in the pathogenesis of lumbar spinal canal stenosis by activating interleukin-6 expression. Eur Spine J 24: 2001-2009, 2015.

18. Gu SX, Li X, Hamilton JL, Chee A, Kca R, Chen D, An HS, Kim JS, Oh CD, Ma YZ, et al: MicroRNA-146a reduces IL-1 dependent inflammatory responses in the intervertebral disc. Gene 555: 80-87, 2015.

19. Hu B, Xu C, Tian Y, Shi C, Zhang Y, Deng L, Zhou H, Cao P, Chen $\mathrm{H}$ and Yuan W: Inflammatory microRNA-194 and-515 attenuate the biosynthesis of chondroitin sulfate during human intervertebral disc degeneration. Oncotarget 8: 49303-49317, 2017.

20. Papadopoulou AS, Serneels L, Achsel T, Mandemakers W, Callaerts-Vegh Z, Dooley J, Lau P, Ayoubi T, Radaelli E, Spinazzi M, et al: Deficiency of the miR-29a/b-1 cluster leads to ataxic features and cerebellar alterations in mice. Neurobiol Di 73: 275-288, 2015.
21. Ranganathan P, Ngankeu A, Zitzer NC, Leoncini P, Yu X, Casadei L, Challagundla K, Reichenbach DK, Garman S, Ruppert AS, et al: Serum miR-29a is upregulated in acute graft-versus-host disease and activates dendritic cells through TLR binding. J Immunol 198: 2500-2512, 2017.

22. Wang WJ, Yu XH, Wang C, Yang W, He WS, Zhang SJ, Yan YG and Zhang J: MMPs and ADAMTSs in intervertebral disc degeneration. Clin Chim Acta 448: 238-246, 2015.

23. Brunet Vega A, Pericay C, Moya I, Ferrer A, Dotor E, Pisa A, Casalots A, Serra-Aracil X, Oliva JC, Ruiz A and Saigí E: microRNA expression profile in stage III colorectal cancer: Circulating miR-18a and miR-29a as promising biomarkers. Oncol Rep 30: 320-326, 2013

24. Roncarati R, Viviani Anselmi C, Losi MA, Papa L, Cavarretta E, Da Costa Martins P, Contaldi C, Saccani Jotti G, Franzone A, Galastri L, et al: Circulating miR-29a, among other up-regulated microRNAs, is the only biomarker for both hypertrophy and fibrosis in patients with hypertrophic cardiomyopathy. J Am Coll Cardiol 63: 920-927, 2014.

This work is licensed under a Creative Commons Attribution-NonCommercial-NoDerivatives 4.0 International (CC BY-NC-ND 4.0) License. 